\title{
Effect of an oral preparation containing hyaluronic acid, chondroitin sulfate, hydrolyzed collagen type II and hydrolyzed keratin on synovial fluid features and clinical indices in knee osteoarthritis. A pilot study
}

\author{
F. Oliviero', R. Ramonda' ${ }^{1}$ A. Hoxha' ${ }^{1}$, A. Scanu ${ }^{1}$, P. Galozzi ${ }^{1}$, M. Favero', \\ P. Frallonardo', L. Punzi ${ }^{2,3}$ \\ ${ }^{1}$ Rheumatology Unit, Department of Medicine - DIMED, University of Padova, Italy; \\ ${ }^{2}$ High Specialty Rehabilitation Hospital, Motta di Livenza (TV), Italy; \\ ${ }^{3}$ Centre for Gout and Metabolic Bone and Joint Diseases, Rheumatology, \\ SS Giovanni and Paolo Hospital, Venice, Italy
}

\section{SUMMARY}

The aim of this study was to evaluate the effect of an oral preparation containing a naturally occurring matrix of hydrolyzed collagen type II, chondroitin sulfate (CS), and hyaluronic acid (HA), and bioactive oligopeptides of natural hydrolyzed keratin $(\mathrm{K})$ in patients affected by knee OA through the evaluation of synovial fluid (SF) and clinical changes before and after treatment.

Thirty patients with knee OA and swollen joint were included in the study and submitted to arthrocentesis. Patients were randomized in two groups:

1) the treatment group (N.15) took a dietary supplement containing $120 \mathrm{mg} \mathrm{HA}, 240 \mathrm{mg} \mathrm{CS}$ and $300 \mathrm{mg} \mathrm{K}$ once a day for 4 weeks;

2) the control group (N.15) was only submitted to arthrocentesis.

Patient symptoms were evaluated at the beginning and at the end of the study by the WOMAC self-assessment questionnaire, the Lequesne algofunctional index, and the VAS forms.

SF changes were evaluated by measuring local inflammatory indices, cytokines IL- $1 \beta$, IL-8, IL-6, IL-10 and GM-CSF. The group of patients treated with the oral supplement showed an improvement in the clinical indices WOMAC $(\mathrm{p}<0.01)$, Lequesne $(\mathrm{p}=0.014)$ and VAS pain $(\mathrm{p}<0.01)$. On the contrary, no significant changes were found in the control group.

The SF collected from the treated group showed a reduction of IL-8 ( $\mathrm{p}=0.015)$, IL-6 and IL-10 levels, while no changes in cytokines were observed in the control group.

This pilot study suggests that an oral administration of a preparation containing a combination of HA, CS and $\mathrm{K}$ can improve some clinical parameters and affect cytokine concentrations in SF in patients with knee OA.

Key words: Osteoarthritis; synovial fluid; hyaluronic acid; chondroitin sulfate; cytokines.

Reumatismo, 2020; 72 (3): 125-130

\section{INTRODUCTION}

steoarthritis $(\mathrm{OA})$ is a chronic disease characterized by articular tissue degeneration and inflammation. Joint pain, short-term stiffness and reduced joint motions are the hallmarks of patients with OA. Disease progression is often severe and can lead to disability. Being the most preva- lent arthropathy worldwide, $\mathrm{OA}$ is also associated to a high social and economic burden (1). The incidence of OA increases with age and, in addition, aged individuals can present different comorbidities which might influence the complexity of the pharmacological treatment.

From the molecular point of view, the mechanisms responsible for OA develop-
Corresponding author: Francesca Oliviero Rheumatology Unit, Department of Medicine - DIMED, University of Padova Via Giustiniani, 2 - 35128 Padova E-mail: francesca.oliviero@unipd.it 
ment have not been yet fully elucidated, but mechanical stress, obesity, trauma and calcium crystals can induce a proinflammatory status in tissue and stimulate a variety of mediators (2). Proteolytic enzymes, cytokines and reactive oxygen species (ROS), in particular, are responsible for the decrease in glycosaminoglycan (GAG) content, which normally maintains articular integrity and function, and for the onset of an inflammatory cascade that sustains the progression of OA. Among GAGs, hyaluronic acid (HA), which is a non-sulfated GAG produced by chondrocytes and synoviocytes, confers to SF important rheological properties and helps to maintain the normal integrity of the joint (3). During OA, HA undergoes depolymerization, thus compromising both the synovial fluid (SF) viscosity and cartilage matrix homeostasis.

To date, there is no cure for OA. Available drugs treat symptoms and most of them have serious side effects. Besides the traditional pharmacological treatment, such as paracetamol and nonsteroidal anti-inflammatory drugs (NSAIDs), the EULAR recommendations for the management of knee OA support the efficacy of symptomatic slow acting drugs (SYSADOA) both for pain reduction and functional improvement (4). Among them, the intraarticular administration of HA has shown to provide beneficial effects in patients with OA $(5,6)$. However, the necessity for multiple injections can cause discomfort in some patients, who, instead, could benefit from an oral administration.

Due to the high incidence of adverse side effects associated with the traditional therapies as well as the increase of the aging population, new safer alternative treatments for OA are definitively needed. In this regard, there is a growing interest in new natural substances with proven efficacy in reducing pain and improving activities of daily living in patients suffering from progressive OA. Among them, emerging evidence is reported for a proprietary nutraceutical grade powder composed of a naturally occurring matrix containing hydrolyzed collagen type
II and low-molecular weight HA and CS. This composition is similar to that of the human articular cartilage in the synovial joints and in previous studies it proved to be safe and effective in reducing Visual Analogic Scale (VAS) and The Western Ontario and McMaster Universities Osteoarthritis Index (WOMAC) pain scores in OA after oral administration (7). A growing attention is also devoted to keratin biopeptides that have been found to be effective in relieving symptoms of knee OA providing strong antioxidant and antiinflammatory activity (8).

The aim of this study was to evaluate the effect of an oral preparation containing a naturally occurring matrix of hydrolyzed collagen type II, chondroitin sulfate (CS), and HA, and Cynatine ${ }^{\circledR}$ FLX, bioactive oligopeptides of natural hydrolyzed keratin $(\mathrm{K})$ in patients affected with knee OA.

\section{MATERIALS AND METHODS}

\section{Patients}

Thirty consecutive patients with knee OA and swollen joint attending the outpatients' clinic of the Rheumatology Unit of the University of Padua (Italy) for arthrocentesis and synovial fluid (SF) analysis were included in the study. All patients were diagnosed according the EULAR criteria for knee OA (9).

Inclusion criteria were: Kellgren-Lawrence grade equal to 2-3, disease duration of 2-5 years, no pharmacological treatment apart from paracetamol, no other concomitant rheumatic disease, no signs of radiologic chondrocalcinosis, a SF effusion higher than $5 \mathrm{ml}$ at baseline and the absence of urate or pyrophosphate crystals in SF. None of the patients had received intra-articular injections for at least 3 months prior to the study onset. We excluded patients who had taken any anti-inflammatory drugs (NSAIDs or corticosteroids) the week before arthrocentesis.

All patients were submitted to arthrocentesis and, after SF collection, were randomized in two groups. The first group (15 patients) were requested to take a dietary supplement containing $120 \mathrm{mg}$ HA, 240 
mg CS, $300 \mathrm{mg} \mathrm{K}$, manganese and piperine (Ialoral ${ }^{\circledR} 1500$, PharmaSuisse Laboratories $\mathrm{Srl}$ ) once a day, every day for 4 weeks. The control group consisted in 15 patients who did not take the supplement and were submitted to arthrocentesis only. After 4 weeks all patients were submitted to SF aspiration only in case of a swollen joint.

Patient symptoms were evaluated at the beginning (T0) and at the end (T4) of the study by the WOMAC self-assessment questionnaire, the Lequesne algofunctional index (Lequesne), and the VAS forms.

Patient data and SF were collected and analyzed following the procedures approved by the local research ethics committee. The study was approved by the Hospital Internal Review Board (Prot. n. 2971P) and carried out according to the principles of the Declaration of Helsinki. All patients gave their written informed consent.

\section{Synovial fluid study}

After arthrocentesis, SF samples were promptly examined for total and differential white blood cell (WBC) count using a Bürker counting chamber and pre-stained slides for cell morphology (Testsimplets $®)$, respectively (10).

SF changes were evaluated through the ELISA assessment of the following cytokines as previously described (11): IL$1 \beta$ (sensitivity $2.34 \mathrm{pg} / \mathrm{mL}), \mathrm{IL}-8(1.20 \mathrm{pg} /$ $\mathrm{mL})$, IL-6 (1.60 pg/mL), IL-10 (2.34 pg/ $\mathrm{mL})$ and $\mathrm{GM}-\mathrm{CSF}(3 \mathrm{pg} / \mathrm{mL})$ (eBioscience, San Diego, California, USA).

\section{Statistical analysis}

The Mann-Whitney test was used to compare the variables between the HA group and the control group at specific time points. The Wilcoxon matched test was used to compare clinical scores and cytokine levels before treatment and the final point.

The statistical analysis was conducted using Graph Pad Prism (Graph Pad Software, Inc.) with a level of significance set at 0.05 .

\section{RESULTS}

The patient characteristics at baseline are outlined in Table I. Age and clinical indices were not different in the two groups before treatment (T0). Changes in the clinical indices are shown in Figure 1. A significant decrease of WOMAC score $(\mathrm{p}<0.01)$, Lequesne $(p=0.014)$ and VAS pain $(p<0.01)$ were observed at 4 weeks in the group of patients treated with the supplement, regardless of the presence/absence of SF. No significant changes were found in the control group (data not shown).

At the end of the treatment, 8 out of the 30 enrolled patients ( 5 in the treatment group and 3 in the control group) did not show evidence of effusion under ultrasound examination and therefore were not submitted to arthrocentesis (T4). The SF volume obtained from the other patients, although less than the initial amount, was greater than $5 \mathrm{ml}$ and, consequently, sufficient for cytokine measurements (mean 12.2 $\mathrm{mL}, \mathrm{SD}$ 8.7). The SF collected from the

Table I - Characteristics of the patients included in the study at baseline.

\begin{tabular}{|c|c|c|c|c|}
\hline & All patients & HA group & CTR group & P value* \\
\hline Age (yrs) & $64.4(6.9)$ & $63.1(9.7)$ & $65.4(4.1)$ & ns \\
\hline WOMAC & $1239.9(292.5)$ & $1250(387.7)$ & $1228.2(306.9)$ & ns \\
\hline LEQ & $13.6(4.6)$ & $14.3(4.3)$ & $12.9(5.1)$ & ns \\
\hline VAS & $67.4(18.5)$ & $72.8(12.8)$ & $62.1(22.1)$ & ns \\
\hline SF-volume (mL) & $18.4(10.7)$ & $19.3(12.9)$ & $16.7(5.5)$ & ns \\
\hline SF-WBC (N. cell/ $/ \mu \mathrm{L})$ & $237.9(202.5)$ & $246.1(234.4)$ & $233.3(175.9)$ & ns \\
\hline SF-PMN (\%) & $3.9(5.4)$ & $3.5(4.3)$ & $4.1(5.8)$ & ns \\
\hline
\end{tabular}

WOMAC, Western Ontario and McMaster Universities OA Index; LEQ, Lequesne index; VAS, visual analog scale; SF, synovial fluid; WBC, white blood cell; PMN, polymorphonucler cells. *Mann-Whitney test between HA and control groups. 
treated group showed a reduction of IL-8 $(\mathrm{p}=0.015)$, IL-6 and IL-10 levels (Figure 2 ), while the concentrations of IL-1ß and GM-CSF were under the detection limits. No SF changes in cytokines were instead observed in the control group: IL- $8=6.2$ $\mathrm{pg} / \mathrm{mL} \pm 8.4$; IL-6 $=254.5 \mathrm{pg} / \mathrm{mL} \pm 303.4$; IL$10=2.6 \mathrm{pg} / \mathrm{mL} \pm 0.6$.

\section{CONCLUSIONS}

Very few pharmacological options are available for OA and most of them are intended to reduce symptoms, such as pain and discomfort. The recent recommendations for the management of knee OA developed by the European society for clinical and economic aspects of osteoporosis, osteoarthritis and musculoskeletal diseases (ESCEO) highlight the importance of the combination of treatment modalities in- cluding non-pharmacological and pharmacological treatments (12). While oral glucosamine sulphate (GS) and CS are strongly recommended for the management of this disease, HA finds a weak consensus only as intra-articular therapy (12, 13).

Contrary to GS and CS, little evidence supports the effectiveness of oral HA in patients with OA. Some randomized, placebo-controlled trials showed an improvement in knee pain and WOMAC score in these patients $(14,15)$. More recently, oral administration of HA has shown beneficial therapeutic effects similar to those obtained by an intra-articular HA injection in patients with knee OA (16).

Instead, a limited number of studies have considered the effect of oral preparations in SF changes. One of that study carried out in patients with knee OA, who were treated

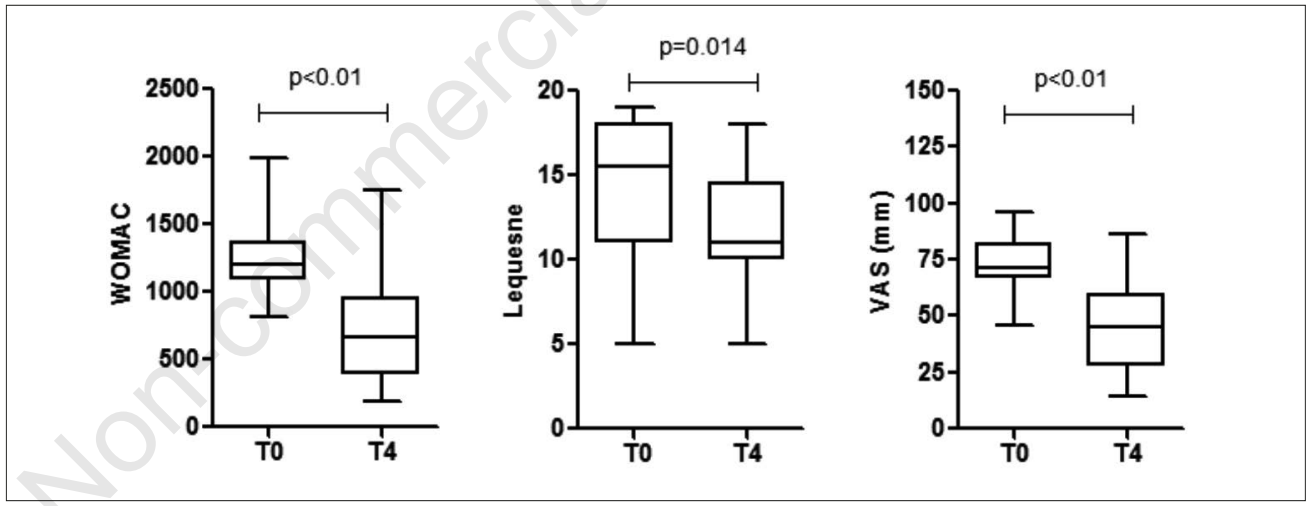

Figure 1 - Changes in clinical indices after 4-week laloral 1500 treatment.
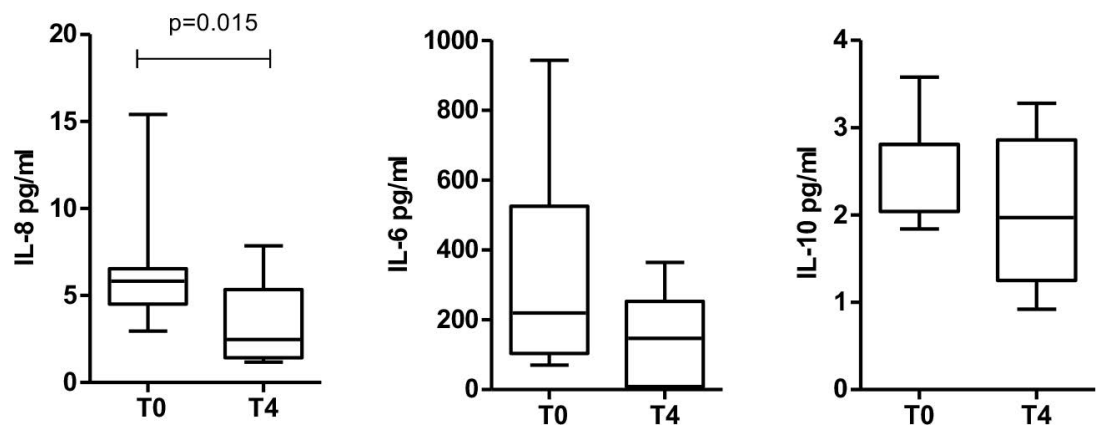

Figure 2 - Changes in IL-8, IL-6 and IL-10 SF levels after 4-week laloral ${ }^{\circledR} 1500$ treatment. 
with oral glucosamine-CS and quercetin combination for 3 months, demonstrated an improvement in SF properties, such as HA molecular size and CS concentration (17). In another study, Nelson et all. tested an oral preparation containing HA in obese patients with OA. After 3 months the patients showed a significant improvement in pain and joint function with a significant decrease in inflammatory cytokines measured both in serum and SF (18).

In our study, patients received a preparation containing both CS and HA, embedded in a highly absorbable matrix of type II hydrolyzed collagen for a relatively short period of time. After a treatment of 4 weeks, patients had actually a significant reduction in clinical indices, such as WOMAC and Lequesne, compared to control subjects. Furthermore, the evaluation of SF before and after treatment showed a decreased cytokine-dependent inflammatory activity, suggesting a potential anti-inflammatory effect of this preparation on the cartilage and the synovial membrane.

Despite the limited number of subjects included in our study, the results are promising especially for those patients who might feel some discomfort due to intra-articular injections. Therefore, this oral supplementation could provide an additional possibility for the treatment of OA.

Further randomized, placebo-controlled trials are needed to establish the efficacy and the mechanism of action of oral HA and CS administrations.

\section{Funding}

This work was supported by a grant from PharmaSuisse Laboratories.

\section{REFERENCES}

1. Hawker GA. Osteoarthritis is a serious disease. Clin Exp Rheumatol. 2019; 37: 3-6.

2. Robinson WH, Lepus CM, Wang Q, et al. Low-grade inflammation as a key mediator of the pathogenesis of osteoarthritis. Nat Rev Rheumatol. 2016; 12: 580-92.

3. Martin-Alarcon L, Schmidt TA. Rheological effects of macromolecular interactions in synovial fluid. Biorheology. 2016; 53: 49-67.

4. Jordan KM, Arden NK, Doherty M, et al. Standing Committee for International Clini- cal Studies Including Therapeutic Trials ESCISIT. EULAR Recommendations 2003: an evidence based approach to the management of knee osteoarthritis: Report of a Task Force of the Standing Committee for International Clinical Studies Including Therapeutic Trials (ESCISIT). Ann Rheum Dis. 2003; 62: 1145-55.

5. Henrotin Y, Raman R, Richette P, et al. Consensus statement on viscosupplementation with hyaluronic acid for the management of osteoarthritis. Semin Arthritis Rheum. 2015; 45: 140-9.

6. Maheu E, Bannuru RR, Herrero-Beaumont $\mathrm{G}$, et al. Why we should definitely include intra-articular hyaluronic acid as a therapeutic option in the management of knee osteoarthritis: Results of an extensive critical literature review. Semin Arthritis Rheum. 2019; 48: 563-72.

7. Schauss AG, Stenehjem J, Park J, et al. Effect of the novel low molecular weight hydrolyzed chicken sternal cartilage extract, biocell collagen, on improving osteoarthritis-related symptoms: a randomized, double-blind, placebocontrolled trial. J Agric Food Chem. 2012; 60: 4096-101.

8. Beer C, Wood S, Veghte RH. A randomized, double-blind, placebo-controlled clinical trial to investigate the effect of Cynatine ${ }^{\circledR}$ FLX on symptoms of osteoarthritis. J Diet Suppl. 2013; 10: 184-94.

9. Ariani A, Manara M, Fioravanti A, et al. The Italian Society for Rheumatology clinical practice guidelines for the diagnosis and management of knee, hip and hand osteoarthritis. Reumatismo. 2019; 71: 5-21.

10. Punzi L. Manuale di analisi del liquido sinoviale. III edizione. Pavia: Edimes editore; 2010.

11. Scanu A, Oliviero F, Ramonda R, et al. Cytokine levels in human synovial fluid during the different stages of acute gout: role of transforming growth factor $\beta 1$ in the resolution phase. Ann Rheum Dis. 2012; 71: 621-4.

12. Bruyère $\mathrm{O}$, Honvo $\mathrm{G}$, Veronese $\mathrm{N}$, et al. An updated algorithm recommendation for the management of knee osteoarthritis from the European Society for Clinical and Economic Aspects of Osteoporosis, Osteoarthritis and Musculoskeletal Diseases (ESCEO). Semin Arthritis Rheum. 2019; 49: 337-50.

13. Bannuru RR, Osani MC, Vaysbrot EE, et al. OARSI guidelines for the non-surgical management of knee, hip, and polyarticular osteoarthritis. Osteoarthr Cartilage. 2019; 27: 1578-89.

14. Tashiro T, Seino S, Sato T, et al. Oral administration of polymer hyaluronic acid alleviates symptoms of knee osteoarthritis: a double-blind, placebo-controlled study over 
a 12-month period. Sci World J. 2012; 2012 : 167928.

15. Kalman DS, Heimer M, Valdeon A, et al. Effect of a natural extract of chicken combs with a high content of hyaluronic acid (Hyal-Joint) on pain relief and quality of life in subjects with knee osteoarthritis: a pilot randomized doubleblind placebo-controlled trial. Nutr J. 2008; 7: 3.

16. Ricci M, Micheloni GM, Berti M, et al. Clinical comparison of oral administration and viscosupplementation of hyaluronic acid (HA) in early knee osteoarthritis. Musculoskelet Surg. 2017; 101: 45-9.
17. Matsuno H, Nakamura H, Katayama K, et al. Effects of an oral administration of glucosamine-chondroitin-quercetin glucoside on the synovial fluid properties in patients with osteoarthritis and rheumatoid arthritis. Biosci Biotechnol Biochem. 2009; 73: 288-92.

18. Nelson FR, Zvirbulis RA, Zonca B, et al. The effects of an oral preparation containing hyaluronic acid (Oralvisc ${ }^{\circledR}$ ) on obese knee osteoarthritis patients determined by pain, function, bradykinin, leptin, inflammatory cytokines, and heavy water analyses. Rheumatol Int. 2015; 35: 43-52. 